\title{
GCU
}

Glasgow Caledonian

University

University for the Common Good

\section{An enhanced photorealistic immersive system using augmented situated visualization within virtual reality}

Insa Iglesias, Maria; Jenkins, Mark; Morison, Gordon

Published in:

2021 IEEE Conference on Virtual Reality and 3D User Interfaces Abstracts and Workshops (VRW)

DOI:

10.1109/VRW52623.2021.00139

Publication date:

2021

Document Version

Author accepted manuscript

Link to publication in ResearchOnline

Citation for published version (Harvard):

Insa Iglesias, M, Jenkins, M \& Morison, G 2021, An enhanced photorealistic immersive system using augmented situated visualization within virtual reality. in 2021 IEEE Conference on Virtual Reality and 3D User Interfaces Abstracts and Workshops (VRW). IEEE, pp. 514-515, 28th IEEE Conference on Virtual Reality and 3D User Interfaces, 27/03/21. https://doi.org/10.1109/VRW52623.2021.00139

\section{General rights}

Copyright and moral rights for the publications made accessible in the public portal are retained by the authors and/or other copyright owners and it is a condition of accessing publications that users recognise and abide by the legal requirements associated with these rights.

Take down policy

If you believe that this document breaches copyright please view our takedown policy at https://edshare.gcu.ac.uk/id/eprint/5179 for details of how to contact us. 


\title{
An Enhanced Photorealistic Immersive System using Augmented Situated Visualization within Virtual Reality
}

\author{
Maria Insa Iglesias* $\quad$ Mark Jenkins $^{\dagger} \quad$ Gordon Morison \\ School of Engineering and Built Environment, Glasgow Caledonian Universit, Glasgow, United Kingdom
}

\begin{abstract}
This work presents a system which allows image data and extracted features from a real-world location to be captured and modelled in a Virtual Reality (VR) environment combined with Augmented Situated Visualizations (ASV) overlaid and registered in a virtual environment. Combining these technologies with techniques from Data Science and Artificial Intelligence (AI)(such as image analysis and 3D reconstruction) allows the creation of a setting where remote locations can be modelled and interacted with from anywhere in the world. This Enhanced Photorealistic Immersive (EPI) system is highly adaptable to a wide range of use cases and users as it can be utilized to model and interact with any environment which can be captured as image data (such as training for operation in hazardous environments, accessibility solutions for exploration of historical/tourism locations and collaborative learning environments). A use case example focused on a structural examination of railway tunnels along with a pilot study is presented, which can demonstrate the usefulness of the EPI system.
\end{abstract}

Keywords: Virtual reality, Situated visualization, Immersive analytics, Visual structural inspection

Index Terms: Human-centered computing-VisualizationVisualization systems and tools-; Computing methodologiesComputer graphics-Interaction paradigms-Graphics systems and interfacesVirtual reality

\section{INTRODUCTION}

Inspecting dangerous environments, such as examination of confined locations, working at height or with chemicals, requires experts taking potentially high health and safety risks. This often requires night long shifts to conduct a manual visual inspection of a target environment, which entails evaluating numerous aspects of each artefact identified from hundreds of artefacts.The time-consuming nature of the method in combination with the risk of inaccuracy due to the subjective nature of human examination, have positioned this industry to automate these services with AI technology. By providing change, anomaly and fault detection within the environment, the information understanding and decision making capability of structural engineers and scientists can be greatly enhanced.

Recreating realistic virtual environments of actual locations has facilitated human immersion in these environments. This provides benefits such as minimizing the risk of workers, reducing cost and enhancing task performance [2]. Similarly, workspaces can be replicated allowing users from around the world to collaborate in the same room, extract insights from large complex datasets and make a decision together [1,3]. Furthermore, one of these environments [3] also integrate AI technology (i.e. Machine Learning processes and Mathematical Modelling) to support domain experts in decisionmaking jointly. Although there are existing AI methods for inspection, experts do not have the AI skills required to extract useful information. The EPI system aims at presenting experts with the

\footnotetext{
*e-mail: maria.insaiglesias@gcu.ac.uk

†e-mail: mark.jenkins@gcu.ac.uk

† e-mail: gordon.morison@gcu.ac.uk
}

output of AI algorithms in a way that they can understand and use, combining a photorealistic VR environment with Augmented Situated visualization (ASV) for automatically detecting and visualising extracted features of a real environment without having to be on-site.

\section{Enhanced Photorealistic Immersive System}

This EPI platform is developed in Unreal Engine 4.19 (UE4.19), a suite of integrated tools for game developers to design and build games, simulations, and visualizations. This system is designed for an HTC Vive HMD with two Vive Controllers or Xbox controller. It has a VR mode and a Desktop mode to make this platform accessible to everyone including those who do not have access to a VR HMD.

The EPI system consist of two virtual scenes: the Immersive Dashboard (see Fig. 2 (a-e)), and the Immersive Photorealistic Environment (see Fig. 2 (f-m)). A case study focused on a structural examination of tunnels demonstrates the effectiveness of the combination of a photorealistic 3D reconstruction of a tunnel [5] in a virtual environment with in situ visualizations of surface defects identified by AI algorithms [6].

\subsection{The Immersive Dashboard}

When a user launches this platform, they are initially presented with a virtual scene with a dashboard, as shown in Figure 2 (a), an environment to allow users to familiarize themselves with the virtual surrounding and visual representation of data. Panels display data using different visualization techniques (e.g. multiple views, road map and, context + overview) for data analysis and extract useful information. Users can interact with the panel by pointing and clicking the panel using the trigger button of controllers. This allows users, for example, to filter and rank data displayed according to a topic of interest. This Immersive Dashboard offers a virtual environment with an interactive panel, facilitating users the exploration of image data without being limited to 2D displays restrictions.

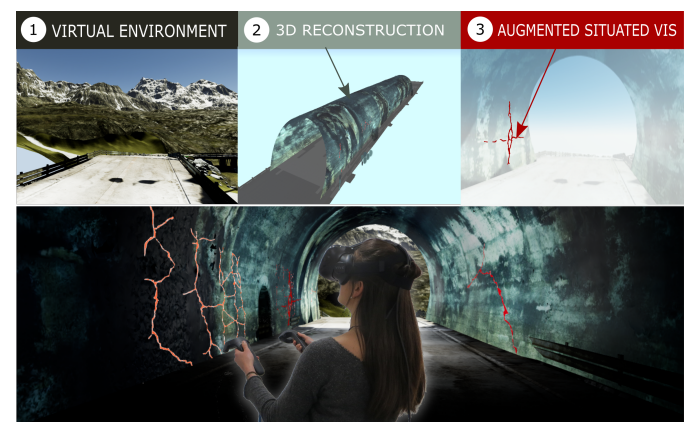

Figure 1: The Development consist of creating the Virtual Environment (1), registering the $3 \mathrm{D}$ reconstruction of the target location (2) before any features are registered (3).

\subsection{The Photorealistic Environment for Data Immersion}

When a user clicks on the left panels shown in Figure 2 (a), they are immersed in the Photorealistic Environment shown in Figure 2 (f) 
where they can move around freely using the touch-pad button of controllers. Users are supported with a set of interactive panels to manage user navigation and ASV rendering. The ASV is displayed when pointing and clicking on data using the trigger button of controllers. Combining the use of both scenes, examiners can interact with the data (i.e. surface defects), evaluate the data (i.e. identify the most severe defects, locations and dimensions), and extract insight (i.e. evaluate structural condition of the tunnel).

\section{Use CASE}

To show feasibility of the system, a use case is presented focused on the use of the EPI system in the structural examination of tunnels (Fig. 1).

System Framework - To develop the EPI system use case, the image dataset that examiners require to analyze is processed following the described pipeline:

1. Unreal Engine Blueprint Visual Scripting (UE4) for VR Environment: This first stage consists of developing a virtual environment using UE4 VR template and open-source 3D assets. Rather than presenting point cloud data with a simple grey background, a real-world environment is simulated to immerse users in the data providing a natural experience.

2. Structure From Motion techniques for 3D Reconstruction: This second stage consists of constructing a 3D model using the Structure From Motion techniques presented in [5]. Then, such a 3D model is imported into the virtual environment and registered in the context scene.

3. AI algorithms for Data Analysis: This stage consist of extracting features using the AI algorithms presented in [6] that have an abstract representation. Using visualization techniques and creating a new class in UE4, the extracted features are registered and overlaid onto the $3 \mathrm{D}$ model.

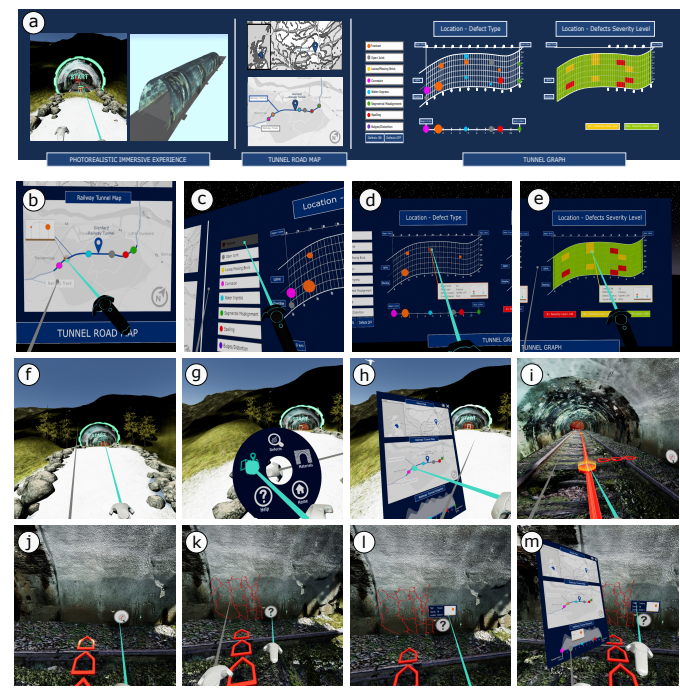

Figure 2: The EPI system consist of the Immersive Dashboard (a-e) and Photorealistic Environment ( $\mathrm{f}-\mathrm{m})$ scene.

Procedure - In the Immersive Dashboard, examiner are immersed in a workspace where they can obtain an overview of the structural condition of the tunnel (see Fig. 2 (b)). Users can explore in detail the defects detected through the interactive panels. For example, there is a road map (see Fig. 2 (b)) that localizes the detected defects geographically and a 2D graph (see Fig. 2(d)) that represents the top view of the tunnel with its defects. The panels on the left (see Fig. 2(c)) allow examiners to filter defects by type, surface material of the tunnel and location, and to rank defects by severity level (see Fig. 2(e)).

In the Photorealistic Environment, users can conduct a realistic structural examination of the tunnel without requiring to be onsite. The watch panel (see Fig. 2(g)), attached to examiners virtual arm, allows the activation of panel tools (see Fig. 2(h)) that support examiners in exploring and evaluating defects. Examiners can either: (i) conduct a throughout structural examination going through the tunnel analyzing each defect in detail identified; or (ii) teletransport to the locations that require a better inspection using the panel tool (Fig. 2(h) shows how an examiner clicks on a specific defect from the map and then the examiner is teletransported to the location (Fig. 2(i)). By clicking on its pin (Fig. 2(j)), Examiners can analyze a defect (Fig. 2(k)) and an informative panel (Fig. 2(m)) is displayed to provide detail information of the defect (e.g. defect location in the tunnel, defect dimension and surface tunnel material).

User Study - In a previous study [4], domain experts showed their attitude toward using an intelligent system for structural inspection, compared to the traditional manual visual inspection. Thus, this work conducted a comparative study to evaluate experts preference toward a desktop version (3D condition) [4] or VR version, approach presented in this paper (VR condition). A total of six professionals took part in the study (average age $=26.2$ years, $s d=$ 1.5) with equal participation of female and male. Participants had to perform the same three tasks in both conditions thinking-out-load, having a different dataset displayed in each condition. All participants completed all tasks successfully in both conditions, having a higher interest and preference to the VR system given the feeling of presence and mental workload (see notes and data analysis in the supplementary material).

\section{Conclusion ANd Future Work}

This paper presents an innovative solution of using visualisation techniques to display extracted features by AI algorithms within 3D photorealistic models of real-world environments, allowing users to remotely analyze a real environment, interact virtually and discover insights without requiring to be on-site. A use case focused on a structural examination of a tunnel is presented that can demonstrates the usefulness of the EPI system, which implements photorealistic VR and superimposed augmented visualizations to evaluate the structural defects identified by AI-based methods. As future work of this work-in-progress system, other use cases beyond tunnels will be carried out on multiple infrastructure types to demonstrate the full power of the system, such as providing accessibility solutions to historical sights and inspecting antique monuments.

\section{REFERENCES}

[1] M. Cavallo, M. Dholakia, M. Havlena, K. Ocheltree, and M. Podlaseck. Dataspace: A reconfigurable hybrid reality environment for collaborative information analysis. In Proc. VR, pp. 145-153. IEEE, 2019.

[2] C. A. Garcia, J. E. Naranjo, F. Gallardo-Cardenas, and M. V. Garcia. Virtual environment for training oil \& gas industry workers. In SALENTO AVR, pp. 379-392. Springer, 2019.

[3] V. Inc. Virtualitics, 2019. https://www.virtualitics.com/, Last accessed on 2019-11-14.

[4] M. Insa-Iglesias, M. Jenkins, and G. Morison. A 3d visual inspection system for structural condition monitoring and analysis. Manuscript submitted for publication, 2020.

[5] M. D. Jenkins, T. Buggy, and G. Morison. An imaging system for visual inspection and structural condition monitoring of railway tunnels. In EESMS, pp. 1-6, 2017.

[6] M. D. Jenkins, T. A. Carr, M. I. Iglesias, T. Buggy, and G. Morison. A deep convolutional neural network for semantic pixel-wise segmentation of road and pavement surface cracks. In Proc. EUSIPCO, pp. 2120-2124, 2018. 